\title{
ON A PROBLEM DUE TO LITTLEWOOD CONCERNING POLYNOMIALS WITH UNIMODULAR COEFFICIENTS
}

\author{
KAI-UWE SCHMIDT
}

\begin{abstract}
Littlewood raised the question of how slowly $\left\|f_{n}\right\|_{4}^{4}-\left\|f_{n}\right\|_{2}^{4}$ (where $\|\cdot\|_{r}$ denotes the $L^{r}$ norm on the unit circle) can grow for a sequence of polynomials $f_{n}$ with unimodular coefficients and increasing degree. The results of this paper are the following. For

$$
g_{n}(z)=\sum_{k=0}^{n-1} e^{\pi i k^{2} / n} z^{k}
$$

the limit of $\left(\left\|g_{n}\right\|_{4}^{4}-\left\|g_{n}\right\|_{2}^{4}\right) /\left\|g_{n}\right\|_{2}^{3}$ is $2 / \pi$, which resolves a mystery due to Littlewood. This is however not the best answer to Littlewood's question: for the polynomials

$$
h_{n}(z)=\sum_{j=0}^{n-1} \sum_{k=0}^{n-1} e^{2 \pi i j k / n} z^{n j+k}
$$

the limit of $\left(\left\|h_{n}\right\|_{4}^{4}-\left\|h_{n}\right\|_{2}^{4}\right) /\left\|h_{n}\right\|_{2}^{3}$ is shown to be $4 / \pi^{2}$. No sequence of polynomials with unimodular coefficients is known that gives a better answer to Littlewood's question. It is an open question as to whether such a sequence of polynomials exists.
\end{abstract}

\section{INTRODUCTION}

For real $r \geq 1$, the $L^{r}$ norm of a polynomial $f \in \mathbb{C}[z]$ on the unit circle is

$$
\|f\|_{r}=\left(\frac{1}{2 \pi} \int_{0}^{2 \pi}\left|f\left(e^{i \theta}\right)\right|^{r} d \theta\right)^{1 / r} .
$$

There is sustained interest in the $L^{r}$ norm of polynomials with restricted coefficients (see, for example, Littlewood [14, Borwein [2], and Erdélyi [5] for surveys on selected problems). Littlewood raised the question of how slowly $\left\|f_{n}\right\|_{4}^{4}-\left\|f_{n}\right\|_{2}^{4}$ can grow for a sequence of polynomials $f_{n}$ with restricted coefficients and increasing degree. This problem is also of interest in the theory of communications, because $\|f\|_{4}^{4}$ equals the sum of squares of the aperiodic autocorrelations of the sequence formed from the coefficients of $f$ [2, p. 122]; in this context one considers the merit factor $\|f\|_{2}^{4} /\left(\|f\|_{4}^{4}-\|f\|_{2}^{4}\right)$. Much work on Littlewood's question has been done when the coefficients are -1 or 1 ; see [8] for recent advances. In the situation where the coefficients are

Date: 13 September 2012 (revised 12 February 2013).

2010 Mathematics Subject Classification. Primary: 42A05, 11B83; Secondary: 94A55. 
restricted to have unit magnitude, the polynomials

$$
g_{n}(z)=\sum_{k=0}^{n-1} e^{\pi i k^{2} / n} z^{k} \quad \text { for integral } n \geq 1
$$

are of particular interest [11, [12, [13, [14, 1] These polynomials are also the main ingredient in Kahane's celebrated semi-probabilistic construction of ultra-flat polynomials [9], which disproves a conjecture due to Erdős [6]. Write

$$
\alpha_{n}=\frac{\left\|g_{n}\right\|_{4}^{4}-\left\|g_{n}\right\|_{2}^{4}}{\left\|g_{n}\right\|_{2}^{3}}
$$

(note that $\|f\|_{2}=\sqrt{n}$ for every polynomial $f$ of degree $n-1$ with unimodular coefficients). Based on the work in [11] and [12] and calculations carried out by Swinnerton-Dyer, Littlewood concluded in [13] that

$$
\lim _{n \rightarrow \infty} \alpha_{n}=\sqrt{2}-\frac{2}{\pi}(\sqrt{2}-1)=1.15051 \ldots,
$$

but expressed doubt in his own conclusion. He knew that

$$
0.604 \leq \alpha_{n} \leq 0.656 \text { for } 18 \leq n \leq 41
$$

and noted [13, Appendix] "There is a considerable mystery here. I have checked my calculations at least six times, and they have been checked also in great detail by Dr. Flett." Littlewood raised this issue again in his book [14, p. 27] and asked for a resolution of this puzzle.

Borwein and Choi [3] conjectured

$$
\left\|g_{n}\right\|_{4}^{4}=n^{2}+\frac{2}{\pi} n^{3 / 2}+\delta_{n} n^{1 / 2}+O\left(n^{-1 / 2}\right),
$$

where $\delta_{n}=-2$ for $n \equiv 0,1(\bmod 4)$ and $\delta_{n}=1$ for $n \equiv 2,3(\bmod 4)$ (this was not stated explicitly as a conjecture in [3], but was confirmed by the authors [4] to be a tentative conclusion based on numerical evidence). This conjecture implies in particular

$$
\lim _{n \rightarrow \infty} \alpha_{n}=\frac{2}{\pi}=0.63661 \ldots
$$

Independently, Antweiler and Bömer [1] made observations similar to (2), while Stańczak and Boche [17] and Mercer [15] derived bounds for $\alpha_{n}$. In particular, Mercer [15] showed that

$$
\limsup _{n \rightarrow \infty} \alpha_{n}<\frac{16}{3 \pi^{3 / 2}}=0.95779 \ldots,
$$

and thereby confirming Littlewood's suspicion (although Mercer was apparently unaware of Littlewood's work).

We shall resolve Littlewood's puzzle by proving that (1) is incorrect and the conjecture (3) is true.

\footnotetext{
${ }^{1}$ Some authors consider $g_{n}\left(e^{ \pm \pi i / n} z\right)$, which however has the same $L^{r}$ norm as $g_{n}(z)$.
} 
Theorem 1. We have

$$
\lim _{n \rightarrow \infty} \frac{\left\|g_{n}\right\|_{4}^{4}-\left\|g_{n}\right\|_{2}^{4}}{\left\|g_{n}\right\|_{2}^{3}}=\frac{2}{\pi}
$$

We shall also show that this is not the best possible answer to Littlewood's question. To do so, we consider the polynomials

$$
h_{n}(z)=\sum_{j=0}^{n-1} \sum_{k=0}^{n-1} e^{2 \pi i j k / n} z^{n j+k} \quad \text { for integral } n \geq 1
$$

of degree $n^{2}-1$, which have been studied by Turyn [18], among others.

Theorem 2. We have

$$
\lim _{n \rightarrow \infty} \frac{\left\|h_{n}\right\|_{4}^{4}-\left\|h_{n}\right\|_{2}^{4}}{\left\|h_{n}\right\|_{2}^{3}}=\frac{4}{\pi^{2}} .
$$

This is the best known answer to Littlewood's question: there is no sequence of polynomials $f_{n}$ with unimodular coefficients for which the limit of $\left(\left\|f_{n}\right\|_{4}^{4}-\left\|f_{n}\right\|_{2}^{4}\right) /\left\|f_{n}\right\|_{2}^{3}$ is known to be less than $4 / \pi^{2}$. It is an open question as to whether such a sequence of polynomials exists.

In the radar literature [10, Ch. 6], the sequences formed from the coefficients of $g_{n}$ and $h_{n}$ are called Chu and Frank sequences, respectively. Our results show that their merit factors grow like $(\pi / 2) \sqrt{n}$ and $\left(\pi^{2} / 4\right) \sqrt{n}$, respectively, which explains numerical results reported in [1].

\section{Proof of Theorem 1}

We begin with summarising known results (see [13, p. 371], for example). For a polynomial $f \in \mathbb{C}[z]$ with $f(z)=\sum_{k=0}^{d-1} a_{k} z^{k}$, we readily verify that

$$
f(z) \overline{f\left(z^{-1}\right)}=\sum_{u=-(d-1)}^{d-1} c_{u} z^{u}
$$

where

$$
c_{u}=\sum_{0 \leq j, j+u<d} a_{j} \overline{a_{j+u}} .
$$

The numbers $c_{u}$ satisfy $c_{u}=\overline{c_{-u}}$. Hence

$$
\|f\|_{4}^{4}=\frac{1}{2 \pi} \int_{0}^{2 \pi}\left(f\left(e^{i \theta}\right) \overline{f\left(e^{i \theta}\right)}\right)^{2} d \theta=c_{0}^{2}+2 \sum_{u=1}^{d-1}\left|c_{u}\right|^{2} .
$$

Lemma 3. For each $n \geq 1$, we have

$$
\left\|g_{n}\right\|_{4}^{4}=n^{2}-\epsilon_{n}+4 \sum_{1 \leq u \leq n / 2}\left(\frac{\sin \left(\pi u^{2} / n\right)}{\sin (\pi u / n)}\right)^{2},
$$

where $\epsilon_{n}=2$ for $n \equiv 2(\bmod 4)$ and $\epsilon_{n}=0$ otherwise. 
Proof. For $f=g_{n}$, elementary manipulations reveal that the numbers $c_{u}$ in (41) satisfy

$$
\left|c_{u}\right|=\left|\frac{\sin \left(\pi u^{2} / n\right)}{\sin (\pi u / n)}\right|
$$

for $1 \leq u \leq n-1$. The desired result then follows from (5) after noting that $c_{0}=n$ and $\left|c_{u}\right|=\left|c_{n-u}\right|$ for $1 \leq u \leq n-1$ and $2\left|c_{n / 2}\right|=\epsilon_{n}$ for even $n$.

We now prove Theorem 1 by finding an asymptotic evaluation of the sum on the right hand side of (6) .

Let $x$ be a real number satisfying $0<x \leq \pi / 2$. From the inequality $x-x^{3} / 6 \leq \sin x \leq x$ we see that

$$
0<\frac{1}{(\sin x)^{2}}-\frac{1}{x^{2}}<1
$$

and therefore

$$
\left|\sum_{1 \leq u \leq n / 2}\left(\frac{\sin \left(\pi u^{2} / n\right)}{\sin (\pi u / n)}\right)^{2}-\sum_{1 \leq u \leq n / 2}\left(\frac{\sin \left(\pi u^{2} / n\right)}{\pi u / n}\right)^{2}\right|<\frac{n}{2} .
$$

Thus, defining the function $r: \mathbb{R} \rightarrow \mathbb{R}$ by

$$
r(x)=\left(\frac{\sin \left(\pi x^{2} / n\right)}{\pi x / n}\right)^{2}
$$

the theorem is proved by showing that

$$
\lim _{n \rightarrow \infty} \frac{1}{n^{3 / 2}} \sum_{1 \leq u \leq n / 2} r(u)=\frac{1}{2 \pi} .
$$

It is consequence of the Euler-Maclaurin formula [16, Theorem B.5] that, for real numbers $a$ and $b$ with $a<b$, the expression

$$
\left|\sum_{a<u \leq b} r(u)-\int_{a}^{b} r(x) d x\right|
$$

is at most

$$
\frac{1}{2}(|r(a)|+|r(b)|)+\frac{1}{12}\left(\left|r^{\prime}(a)\right|+\left|r^{\prime}(b)\right|+\int_{a}^{b}\left|r^{\prime \prime}(x)\right| d x\right) .
$$

We take $b=n / 2$ and let $a$ tend to zero. Elementary calculus shows that

$$
|r(n / 2)| \leq \frac{4}{\pi^{2}}, \quad\left|r^{\prime}(n / 2)\right| \leq \frac{8}{\pi}+\frac{16}{n \pi^{2}}, \quad \lim _{a \rightarrow 0} r(a)=\lim _{a \rightarrow 0} r^{\prime}(a)=0,
$$

and $\left|r^{\prime \prime}(x)\right| \leq 34$ for all real $x$. Therefore

$$
\left|\sum_{1 \leq u \leq n / 2} r(u)-\int_{0}^{n / 2} r(x) d x\right| \leq \frac{2}{\pi^{2}}+\frac{2}{3 \pi}+\frac{4}{3 n \pi^{2}}+\frac{17 n}{12},
$$


and so

$$
\lim _{n \rightarrow \infty} \frac{1}{n^{3 / 2}} \sum_{1 \leq u \leq n / 2} r(u)=\lim _{n \rightarrow \infty} \frac{1}{n^{3 / 2}} \int_{0}^{n / 2} r(x) d x,
$$

provided that both limits exist. Substituting $y=\pi x^{2} / n$, we see that this last expression equals

$$
\lim _{n \rightarrow \infty} \frac{1}{2 \pi^{3 / 2}} \int_{0}^{\pi n / 4} \frac{(\sin y)^{2}}{y^{3 / 2}} d y=\frac{1}{2 \pi^{3 / 2}} \int_{0}^{\infty} \frac{(\sin y)^{2}}{y^{3 / 2}} d y .
$$

This establishes (7), and so completes the proof, since

$$
\int_{0}^{\infty} \frac{(\sin y)^{2}}{y^{3 / 2}} d y=\sqrt{\pi}
$$

(see Gradshteyn and Ryzhik [7, 3.823]).

For completeness, we sketch a proof of the identity (8). To do so, we readily verify that

$$
\frac{\Gamma(3 / 2)}{y^{3 / 2}}=\int_{0}^{\infty} e^{-y t} \sqrt{t} d t \quad \text { for } y>0,
$$

which together with $\Gamma(3 / 2)=\sqrt{\pi} / 2$ yields

$$
\int_{0}^{\infty} \frac{(\sin y)^{2}}{y^{3 / 2}} d y=\frac{2}{\sqrt{\pi}} \int_{0}^{\infty} \int_{0}^{\infty} e^{-y t} \sqrt{t}(\sin y)^{2} d t d y .
$$

Since the integrand on the right hand side is nonnegative, we can interchange the order of integration by Tonelli's theorem. The integral therefore equals

$$
\frac{2}{\sqrt{\pi}} \int_{0}^{\infty} \sqrt{t} \int_{0}^{\infty} e^{-y t}(\sin y)^{2} d y d t=\frac{2}{\sqrt{\pi}} \int_{0}^{\infty} \frac{2 \sqrt{t}}{t^{3}+4 t} d t=\sqrt{\pi} .
$$

The inner integral on the left hand side is just the Laplace transform of $(\sin y)^{2}$, while the integral on the right hand side can be evaluated by first substituting $t=x^{2}$ (which makes the integrand rational) and then using standard techniques.

\section{Proof of Theorem 2}

We begin with proving a counterpart of Lemma 3 for the polynomials $h_{n}$.

Lemma 4. For each $n \geq 1$, we have

$$
\left\|h_{n}\right\|_{4}^{4}=n^{4}-\gamma_{n}+8 n \sum_{1 \leq v \leq n / 2} \sum_{1 \leq k \leq v}\left(\frac{\sin (\pi k / n)}{\sin (\pi v / n)}\right)^{2},
$$

where

$$
\gamma_{n}= \begin{cases}3 n^{2} & \text { for even } n \\ 2 n^{2}-2 n & \text { for odd } n\end{cases}
$$


Proof. Write $\zeta=e^{2 \pi i / n}$. Then, for $f=h_{n}$, the numbers $c_{u}$ in (4) are given by (see also Turyn [18])

$$
c_{n u+v}=\sum_{j=0}^{n-u-1} \sum_{k=0}^{n-v-1} \zeta^{j k-(j+u)(k+v)}+\sum_{j=0}^{n-u-2} \sum_{k=n-v}^{n-1} \zeta^{j k-(j+u+1)(k+v)}
$$

for $0 \leq u, v<n$. Rearrange and use $\sum_{k=0}^{n-1} \zeta^{k(u+1)}=0$ for $n \nmid u+1$ (note that the second term is zero for $u+1=n$ ) to see that

$$
\overline{c_{n u+v}}=\zeta^{u v} \sum_{k=0}^{n-v-1} \zeta^{k u} \sum_{j=0}^{n-u-1} \zeta^{j v}-\zeta^{(u+1) v} \sum_{k=0}^{n-v-1} \zeta^{k(u+1)} \sum_{j=0}^{n-u-2} \zeta^{j v}
$$

for $0 \leq u, v<n$. Evaluation of the sums over $j$ gives, for $0 \leq u<n$ and $0<v<n$,

$$
\begin{aligned}
\overline{c_{n u+v}} & =\frac{1}{\zeta^{v}-1} \sum_{k=0}^{n-v-1}\left(\zeta^{k u}\left(1-\zeta^{u v}\right)-\zeta^{k(u+1)}\left(1-\zeta^{(u+1) v}\right)\right) \\
& =\frac{1}{\zeta^{v}-1} \sum_{k=0}^{n-v-1}\left[\zeta^{(k+v) u}\left(\zeta^{k+v}-1\right)-\zeta^{k u}\left(\zeta^{k}-1\right)\right] .
\end{aligned}
$$

We can write this as

$$
\left(\sum_{k=v}^{n-1}-\sum_{k=0}^{n-v-1}\right) \zeta^{k u} \frac{\zeta^{k}-1}{\zeta^{v}-1}
$$

from which we see that

$$
\sum_{u=0}^{n-1}\left|c_{n u+v}\right|^{2}=n\left(\sum_{k=v}^{n-1}+\sum_{k=0}^{n-v-1}-\sum_{k=v}^{n-v-1}-\sum_{k=v}^{n-v-1}\right)\left|\frac{\zeta^{k}-1}{\zeta^{v}-1}\right|^{2}
$$

for $0<v<n$. For $0<v<n / 2$ all of these sums are nonempty, so that after grouping them together we have, for $0<v<n / 2$,

$$
\begin{aligned}
\sum_{u=0}^{n-1}\left|c_{n u+v}\right|^{2} & =n\left(\sum_{k=n-v}^{n-1}+\sum_{k=0}^{v-1}\right)\left|\frac{\zeta^{k}-1}{\zeta^{v}-1}\right|^{2} \\
& =2 n \sum_{k=0}^{v}\left|\frac{\zeta^{k}-1}{\zeta^{v}-1}\right|^{2}-n \\
& =2 n \sum_{k=1}^{v}\left(\frac{\sin (\pi k / n)}{\sin (\pi v / n)}\right)^{2}-n .
\end{aligned}
$$

Using (9) we readily verify that $c_{n u}=0$ for $u \neq 0$. Therefore, since $c_{0}=n^{2}$ trivially, we have from (5)

$$
\left\|h_{n}\right\|_{4}^{4}=n^{4}+2 \sum_{v=1}^{n-1} \sum_{u=0}^{n-1}\left|c_{n u+v}\right|^{2} .
$$


We also have

$$
c_{n u+v}=-\zeta^{v} c_{n u+n-v} \text { for }(u, v) \neq(0,0),
$$

which also follows from (9) using the identities

$$
\sum_{k=0}^{v-1} \zeta^{k w}=-\zeta^{w v} \sum_{k=0}^{n-v-1} \zeta^{k w}
$$

for integers $w$ and $v$ satisfying $n \nmid w$ and $0 \leq v<n$ and

$$
\sum_{j=0}^{n-w-1} \zeta^{-j v}=\zeta^{(w+1) v} \sum_{j=0}^{n-w-1} \zeta^{j v}
$$

for integers $w$ and $v$.

Now, for odd $n$, we have from (12) and (13)

$$
\left\|h_{n}\right\|_{4}^{4}=n^{4}+4 \sum_{v=1}^{(n-1) / 2} \sum_{u=0}^{n-1}\left|c_{n u+v}\right|^{2}
$$

and the desired result follows from (11). Similarly, for even $n$, we have

$$
\left\|h_{n}\right\|_{4}^{4}=n^{4}+4 \sum_{v=1}^{n / 2-1} \sum_{u=0}^{n-1}\left|c_{n u+v}\right|^{2}+2 \sum_{u=0}^{n-1}\left|c_{n u+n / 2}\right|^{2} .
$$

Using (10), we find that

$$
2 \sum_{u=0}^{n-1}\left|c_{n u+n / 2}\right|^{2}=\frac{n}{2} \sum_{k=0}^{n-1}\left|\zeta^{k}-1\right|^{2}=n^{2}
$$

and therefore, by (11),

$$
\left\|h_{n}\right\|_{4}^{4}=n^{4}-n^{2}+4 n+8 n \sum_{v=1}^{n / 2-1} \sum_{k=1}^{v}\left(\frac{\sin (\pi k / n)}{\sin (\pi v / n)}\right)^{2} .
$$

To obtain the desired expression in the lemma for even $n$, we extend the summation over $v$ to $n / 2$ and subtract the correction term

$$
8 n \sum_{k=1}^{n / 2}(\sin (\pi k / n))^{2}=n \sum_{k=0}^{n-1}\left|\zeta^{k}-1\right|^{2}+4 n=2 n^{2}+4 n .
$$

In order to prove Theorem 2, we invoke Lemma 4 and show that

$$
8 n \sum_{1 \leq v \leq n / 2} \sum_{1 \leq k \leq v}\left(\frac{\sin (\pi k / n)}{\sin (\pi v / n)}\right)^{2}=\frac{4}{\pi^{2}} n^{3}+O\left(n^{2}\right) .
$$

To do so, we make repeated use of the following elementary bound, which is also a simple consequence of the Euler-Maclaurin formula [16, Theorem B.5]. 
Let $r: \mathbb{R} \rightarrow \mathbb{R}$ be a differentiable function and let $a$ and $b$ be real numbers with $a<b$. Then

$$
\left|\sum_{a<k \leq b} r(k)-\int_{a}^{b} r(x) d x\right| \leq \frac{1}{2}\left(|r(a)|+|r(b)|+\int_{a}^{b}\left|r^{\prime}(x)\right| d x\right) .
$$

We first take $r(x)=(\sin (\pi x / n))^{2}$ and $(a, b)=(0, v)$, so that for $1 \leq v \leq n / 2$, we have

$$
\begin{aligned}
\sum_{k=1}^{v}(\sin (\pi k / n))^{2} & =\int_{0}^{v}(\sin (\pi x / n))^{2} d x+O(1) \\
& =\frac{n}{\pi} \int_{0}^{\pi v / n}(\sin y)^{2} d y+O(1) \\
& =\frac{n}{2 \pi}(\pi v / n-\sin (\pi v / n) \cos (\pi v / n))+O(1) .
\end{aligned}
$$

Letting

we then have

$$
p(y)=\frac{y-\sin y \cos y}{(\sin y)^{2}}
$$

$$
\sum_{1 \leq v \leq n / 2} \sum_{1 \leq k \leq v}\left(\frac{\sin (\pi k / n)}{\sin (\pi v / n)}\right)^{2}=\frac{n}{2 \pi} \sum_{1 \leq v \leq n / 2} p(\pi v / n)+O(n) .
$$

We now apply (15) with $r(x)=p(\pi x / n)$ and $b=n / 2$ and let $a$ tend to zero. We have

$$
p^{\prime}(y)=2-\frac{2(y-\sin y \cos y) \cos y}{(\sin y)^{3}}
$$

from which, using $x-x^{3} / 6 \leq \sin x \leq x$ and $1-x^{2} / 2 \leq \cos x \leq 1$ together with elementary calculus, we find that

$$
-3<p^{\prime}(y) \leq 2 \text { for } 0<y \leq \pi / 2 .
$$

Hence $\left|r^{\prime}(x)\right|<3 \pi / n$ for $0<x \leq n / 2$. Since we also have $r(n / 2)=\pi / 2$ and $\lim _{a \rightarrow 0} r(a)=0$, we find from (15) that (16) equals

$$
\frac{n}{2 \pi} \int_{0}^{n / 2} p(\pi x / n) d x+O(n)=\frac{n^{2}}{2 \pi^{2}} \int_{0}^{\pi / 2} p(y) d y+O(n) .
$$

The desired result (14) is then established by showing that

$$
\int_{0}^{\pi / 2} p(y) d y=1
$$

By differentiation we readily verify that

$$
\int \frac{y-\sin y \cos y}{(\sin y)^{2}} d y=-\frac{y}{\tan y}+C
$$

for some arbitrary constant $C$ and (17) follows by application of l'Hôpital's rule. 


\section{REFERENCES}

[1] M. Antweiler and L. Bömer. Merit factor of Chu and Frank sequences. IEE Electron. Lett., 46(25):2068-2070, 1990.

[2] P. Borwein. Computational Excursions in Analysis and Number Theory. CMS Books in Mathematics. Springer-Verlag, New York, NY, 2002.

[3] P. Borwein and K.-K. S. Choi. Merit factors of character polynomials. J. London Math. Soc., 61:706-720, 2000.

[4] P. Borwein and K.-K. S. Choi. Personal communication, 2012.

[5] T. Erdélyi. Polynomials with Littlewood-type coefficient constraints. In Approximation theory, X (St. Louis, MO, 2001), Innov. Appl. Math., pages 153-196. Vanderbilt Univ. Press, Nashville, TN, 2002.

[6] P. Erdős. An inequality for the maximum of trigonometric polynomials. Ann. Polon. Math., 12:151-154, 1962.

[7] I. S. Gradshteyn and I. M. Ryzhik. Table of integrals, series, and products. Elsevier/Academic Press, Amsterdam, 7th edition, 2007.

[8] J. Jedwab, D. J. Katz, and K.-U. Schmidt. Littlewood polynomials with small $L^{4}$ norm, 2012. arXiv:1205.0260v1 [math.NT].

[9] J. P. Kahane. Sur les polynômes à coefficients unimodulaires. Bull. London Math. Soc., 12:321-342, 1980.

[10] N. Levanon and E. Mozeson. Radar signals. Wiley-Interscience, 1st edition, 2004.

[11] J. E. Littlewood. On the mean values of certain trigonometric polynomials. J. London Math. Soc., 36:307-334, 1961.

[12] J. E. Littlewood. On the mean values of certain trigonometric polynomials II. Illinois J. Math., 6:1-39, 1962.

[13] J. E. Littlewood. On polynomials $\sum^{n} \pm z^{m}, \sum^{n} e^{\alpha_{m} i} z^{m}, z=e^{\theta i}$. J. London Math. Soc., 41:367-376, 1966.

[14] J. E. Littlewood. Some Problems in Real and Complex Analysis. Heath Mathematical Monographs. D. C. Heath and Company, Lexington, MA, 1968.

[15] I. D. Mercer. Bounds on asymptotic merit factor of Chu sequences, 2012. http://www.math.udel.edu/ idmercer/publications.html.

[16] H. L. Montgomery and R. C. Vaughan. Multiplicative number theory. I. Classical theory, volume 97 of Cambridge Studies in Advanced Mathematics. Cambridge University Press, Cambridge, 2007.

[17] S. Stańczak and H. Boche. Aperiodic properties of generalized binary Rudin-Shapiro sequences and some recent results on sequences with a quadratic phase function. In Proc. of International Zurich Seminar on Broadband Communications, pages 279286. IEEE, 2000.

[18] R. Turyn. The correlation function of a sequences of roots of 1. IEEE Trans. Inf. Theory, 13(3):524-525, 1967.

Faculty of Mathematics, Otto-Von-Guericke University, Universitätsplatz 2, 39106 Magdeburg, Germany.

E-mail address: kaiuwe.schmidt@ovgu.de 